\title{
Detection of influenza viruses by coupling multiplex reverse-transcription loop-mediated isothermal amplification with cascade invasive reaction using nanoparticles as a sensor
}

\author{
This article was published in the following Dove Press journal: \\ International Journal of Nanomedicine \\ 3 April 2017 \\ Number of times this article has been viewed
}

Yiyue $\mathrm{Ge}^{\mathrm{l}}$

Qiang Zhou

Kangchen Zhao'

Ying $\mathrm{Chi}^{\mathrm{I}}$

Bin $\mathrm{Liu}^{3}$

Xiaoyan $\mathrm{Min}^{4}$

Zhiyang Shi'

Bingjie Zou ${ }^{2}$

Lunbiao Cui'

'Institute of Pathogenic Microbiology, Key Laboratories of Enteric

Pathogenic Microbiology (Ministry of Health), Jiangsu Provincial Center for Disease Control and Prevention, ${ }^{2}$ Department of Pharmacology, Jinling Hospital, Medical School of Nanjing University, ${ }^{3}$ Department of Biomedical Engineering, Nanjing Medical University, ${ }^{4}$ Department of Geriatrics, The First Affiliated Hospital of Nanjing Medical University, Nanjing, Jiangsu, People's Republic of China

Correspondence: Lunbiao Cui Institute of Pathogenic Microbiology, Jiangsu Provincial Center for Disease Control and Prevention, No 172 Jiangsu

Road, Nanjing, Jiangsu 210009,

People's Republic of China

Tel +862583759424

Email lbcui@jscdc.cn

Bingjie Zou

Department of Pharmacology, Jinling Hospital, Medical School of Nanjing University, No 305 Zhongshan East

Road, Nanjing, Jiangsu 210002,

People's Republic of China

Tel +862580860196

Email zbj523@I63.com

\begin{abstract}
Influenza virus infections represent a worldwide public health and economic problem due to the significant morbidity and mortality caused by seasonal epidemics and pandemics. Sensitive and convenient methodologies for detection of influenza viruses are essential for further disease control. Loop-mediated isothermal amplification (LAMP) is the most commonly used method of nucleic acid isothermal amplification. However, with regard to multiplex LAMP, differentiating the ladder-like LAMP products derived from multiple targets is still challenging today. The requirement of specialized instruments has further hindered the on-site application of multiplex LAMP. We have developed an integrated assay coupling multiplex reverse transcription LAMP with cascade invasive reaction using nanoparticles (mRT-LAMP-CIRN) as a sensor for the detection of three subtypes of influenza viruses: A/H1N1pdm09, A/H3 and influenza B. The analytic sensitivities of the mRT-LAMP-CIRN assay were $10^{1}$ copies of RNA for both $\mathrm{A} / \mathrm{H} 1 \mathrm{~N} 1 \mathrm{pdm} 09$ and $\mathrm{A} / \mathrm{H} 3$, and $10^{2}$ copies of RNA for influenza B. This assay demonstrated highly specific detection of target viruses and could differentiate them from other genetically or clinically related viruses. Clinical specimen analysis showed the mRT-LAMP-CIRN assay had an overall sensitivity and specificity of $98.3 \%$ and $100 \%$, respectively. In summary, the mRT-LAMP-CIRN assay is highly sensitive and specific, and can be used as a cost-saving and instrument-free method for the detection of influenza viruses, especially for on-site use.
\end{abstract}

Keywords: multiplex, LAMP, gold nanoparticles, on-site detection, influenza virus

\section{Introduction}

Influenza viruses pose an ongoing threat to human health principally through their ability to cause acute respiratory diseases worldwide. ${ }^{1,2}$ Influenza virus evolves rapidly by reassortment and genetic drift and is one of the most common causes of morbidity and mortality, leading to a significant economic impact every year., According to the World Health Organization (WHO), seasonal influenza infections result in $~ 3-5$ million severe cases and 300,000-500,000 deaths around the world yearly. ${ }^{5} \mathrm{~A}$ rapid and sensitive methodology for the early detection of seasonal influenza viruses is of paramount importance to facilitate clinical care, infection control, as well as epidemiologic investigations. Among the diagnostic tools, nucleic acid-based tests are more rapid and sensitive than traditional techniques such as serological assays and influenza virus isolation. At present, real-time reverse transcription polymerase chain reaction (RT-PCR) is a powerful molecular diagnostic method for influenza 
virus infections. ${ }^{6-9}$ In contrast to PCR-based target gene detection, which requires bulky and expensive equipments and highly skilled technicians, recent research has focused on the development of isothermal amplification methods for simple and user-friendly pathogen detection. As the reaction is conducted under isothermal conditions, the thermal cycler is not required, which makes these methods more suitable for use in resource-limited regions or for field use.

As the most commonly used method of isothermal amplification, loop-mediated isothermal amplification (LAMP) allows amplification of DNA with high sensitivity and specificity. ${ }^{10,11}$ LAMP can also be used to detect RNA template by simply adding a reverse transcriptase enzyme under identical reaction conditions as LAMP. ${ }^{10,12}$ To date, RT-LAMP methods have been developed to detect various RNA viruses, including influenza viruses. ${ }^{13-17}$ Multiplex detection represents the development trend of molecular diagnostic methods due to the properties of cost- and time-saving. Although previous studies have described several multiplex LAMP methods, how to differentiate the ladder-like LAMP products derived from multiple targets is still challenging. These methods used either end-point (pyrosequencing, ${ }^{18}$ gel electrophoresis, ${ }^{19}$ etc) or real-time (annealing curve, ${ }^{20}$ fluorescence technology, ${ }^{21,22}$ etc) analysis to differentiate the multiple target sequences. The main drawback of these strategies is the requirement of complicated and specialized instruments, which hinders the on-site use of multiplex LAMP.

To take full advantage of multiplex LAMP, the product identification methodology should be simple and costeffective. Hence, the colorimetric detection would be ideal due to its simplicity, user-friendliness, and ease to interpret the result. Utilization of oligonucleotide probe-modified gold nanoparticles (AuNPs), the aggregation of which can cause notable changes in optical property, ${ }^{23-25}$ seems to satisfy this condition. In the present study, we developed a multiplex RT-LAMP (mRT-LAMP) assay for simultaneous amplification of three subtypes of influenza viruses (influenza A/H1N1pdm09, A/H3 and influenza B), and the RT-LAMP amplicons were further identified by cascade invasive reaction ${ }^{26,27}$ using oligonucleotide probe-modified AuNPs as a sensor. The results can be observed and determined easily based on color changes by naked eyes. mRT-LAMP coupled with cascade invasive reaction using nanoparticles as a sensor (mRT-LAMP-CIRN) provides us a sensitive, specific, cost-saving and instrument-free diagnostic tool for the identification of influenza viruses, especially in resourcelimited settings.

\section{Materials and methods Viral isolates and clinical specimens}

Three influenza virus strains A/Jiangsu/2/2009(H1N1), A/Nanjing/1/2009(H3N2) and B/Jiangsu/01/2015 isolated from patient specimens at Jiangsu Provincial Center for Disease Control and Prevention were used for the development of mRT-LAMP-CIRN assay. Eight clinically or genetically related viruses were used as control to evaluate the specificity of the assay, including seasonal influenza virus $\mathrm{A} / \mathrm{H} 1 \mathrm{~N} 1$, avian influenza viruses $\mathrm{A} / \mathrm{H} 5 \mathrm{~N} 1, \mathrm{~A} / \mathrm{H} 7 \mathrm{~N} 9$ and $\mathrm{A} / \mathrm{H} 9 \mathrm{~N} 2$, respiratory syncytial viruses types $\mathrm{A}$ and $\mathrm{B}$, and parainfluenza viruses types 1 and 2 . A total of 94 nasopharyngeal swab specimens collected from influenza-like cases, including 58 known influenza-positive specimens (35 A/H1N1pdm09, 14 A/H3N2, and nine influenza $B$ ), were used to validate the mRT-LAMPCIRN assay. All the isolates and specimens were kept at $-80^{\circ} \mathrm{C}$ until use. Written informed consent regarding the use of clinical specimens for investigational purposes was obtained from all the patients involved. This study was approved by the Ethics Committee of the Jiangsu Provincial Center for Disease Control and Prevention. All the experiments were carried out in accordance with the ethical guidelines for medical and health research involving human subjects.

\section{Isolation of viral RNA}

Viral RNA was extracted from $200 \mu \mathrm{L}$ of specimens with a High Pure viral RNA kit (Roche Diagnostics, Manheim, Germany) according to the manufacturer's instructions. Purified RNA was eluted with $50 \mu \mathrm{L}$ of elution buffer $(\mathrm{pH}=8.0)$ and kept at $-80^{\circ} \mathrm{C}$ until further analysis.

\section{Preparation of RNA standards}

The hemagglutinin $(H A)$ gene, matrix protein $1(M 1)$ gene and nonstructural protein 1 (NS1) gene were amplified from the influenza viruses A/Jiangsu/2/2009(H1N1), A/Nanjing/1/2009(H3N2) and B/Jiangsu/01/2015 with primers containing $\mathrm{T} 7$ promoter sequence on reverse sides. RNA was transcribed directly from purified PCR amplicons containing the full-length region of each target for mRTLAMP by the use of T7 RNA polymerase (TaKaRa Biotechnology Co. Ltd., Dalian, People's Republic of China). The synthetic RNA transcripts were purified and quantified by reading absorbance at $A_{260 \mathrm{~nm}},{ }^{28}$ and then ten-fold diluted as RNA standards ranging from $10^{4}$ to $10^{\circ}$ RNA copies $/ \mu \mathrm{L}$.

\section{Design of primers and probes}

mRT-LAMP primers were designed based on the conserved regions of $H A$ gene, $M 1$ gene and NS1 gene for influenza 
A/H1N1pdm09, A/H3 and influenza B, respectively. RTLAMP primers were designed using a PrimerExplorer version 4 program on the Eiken website (http://primerexplorer. $\mathrm{jp} / \mathrm{e} /$ ). A set of six primers, including two outer primers (F3/B3), two inner primers (FIP/BIP), and two loop primers (LF/LB), were selected for each of the three gene targets. The specificity and feasibility of the selected primers were subsequently checked by Basic Local Alignment Search Tool search against National Center for Biotechnology Information database. The sequence information of the selected final mRT-LAMP primers containing degenerate bases is shown in Table 1, and the details of the primers are shown in Figure 1.

Using the Universal Invader Design Software version 1.2.4 (Third Wave Technologies, Inc., Madison, WI, USA), three sets of cascade invasive reaction upstream and downstream probes were designed based on the sequences of mRTLAMP amplicons. The sequence information of the selected upstream, downstream and hairpin probes used in secondary invasive reaction, as well as two types of oligonucleotide probes (nanoparticle probes) used for modification of AuNPs is shown in Table 2. All the primers and probes were synthesized and modified by TaKaRa Biotechnology Co. Ltd.

\section{Preparation and modification of AuNPs}

AuNPs were synthesized by reducing tetrachloroauric acid with trisodium citrate, and then modified with oligonucleotide probes according to the protocol described previously. ${ }^{29}$ Two types of nanoparticle probes (Table 2), which were complementary to the hairpin probe, were used to modify the two batches of AuNPs separately. Transmission electron microscopy (TEM) (JEM 2100F; JEOL Co. Ltd., Tokyo, Japan) was used to measure the sizes of oligonucleotide probe-modified AuNPs, which resulted in an average diameter of $13 \mathrm{~nm}$. The ultraviolet (UV) spectrum and TEM images of oligonucleotide probe-modified AuNPs are described in detail in the Supplementary material.

\section{mRT-LAMP reaction}

mRT-LAMP reaction was carried out using the RNA Amplification Kit (Eiken China Co., Ltd., Shanghai, People's Republic of China) in a final volume of $20 \mu \mathrm{L}$ containing $10 \mu \mathrm{L}$ of Reaction Mix, three sets of primers for influenza A/H1N1pdm09, A/H3 and influenza B $(0.15 \mu \mathrm{M}$ each of F3 and B3 primers, $1.2 \mu \mathrm{M}$ each of FIP and BIP primers and $0.6 \mu \mathrm{M}$ each of LF and LB primers), $0.8 \mu \mathrm{L}$ of Enzyme Mix and $1 \mu \mathrm{L}$ of RNA template. Distilled water was used as

Table I Primers used for multiplex RT-LAMP assay

\begin{tabular}{|c|c|c|c|c|}
\hline Target & Name & Type & Sequence $\left(5^{\prime}-3^{\prime}\right)$ & Length (nt) \\
\hline \multirow[t]{8}{*}{ A/HINIpdm09, (HA gene) } & 09HI-F3 & Forward outer & CCGGGAGACAAAATAACATTC & 21 \\
\hline & 09HI-B3 & Reverse outer & GTATATTCTGAAATGGGAGGC & 21 \\
\hline & 09HI-FIB & Forward inner & CAGATCCAGCATTTCTTTCCATTGGAAG & 45 \\
\hline & & & CAACTGGAAATCTAGTG(FIC+F2) & \\
\hline & 09HI-BIP & Reverse inner & TATCATTTCAGATACACCAGTCCACTGG & 45 \\
\hline & & & TGTTTATAGCACCCTTG(BIC+B2) & \\
\hline & 09HI-LF & Forward loop & CGAATGCATATCTCGGYAC & 19 \\
\hline & $09 \mathrm{HI}-\mathrm{LB}$ & Reverse loop & ATACAACTTGTCARACACC & 19 \\
\hline \multirow[t]{8}{*}{$\mathrm{A} / \mathrm{H} 3,(\mathrm{M} /$ gene $)$} & H3-F3 & Forward outer & AGGATGGGGGCTGTAACC & 18 \\
\hline & H3-B3 & Reverse outer & CCAGCCATTTGCTCCATAGC & 20 \\
\hline & H3-FIB & Forward inner & TGAGACCTGTGCTGGGAGTCAGGTG & 40 \\
\hline & & & GCMTTTGGCCTGGTA & \\
\hline & H3-BIP & Reverse inner & TAGGCARATGGTGGCAACAACCTGT & $4 I$ \\
\hline & & & AGTGCTGGCCARAACC & \\
\hline & H3-LF & Forward loop & AATCTGYTCACATGTTGCACA & 21 \\
\hline & H3-LB & Reverse loop & CATTAATAARACATGAGAACAGAAT & 25 \\
\hline \multirow[t]{8}{*}{ Influenza B, (NSI gene) } & FluB-F3 & Forward outer & AGGGACATGAACAACAAAGA & 20 \\
\hline & FluB-B3 & Reverse outer & CAAGTTTAGCAACAAGCCT & 19 \\
\hline & FluB-FIB & Forward inner & TMARGGACAATACATTACGCATATC & 49 \\
\hline & & & GATAAAGGAGGAAGTAAACACTCA & \\
\hline & FluB-BIP & Reverse inner & TAAAYGGAACATTCCTCAAACACCACTC & 43 \\
\hline & & & TGGTCATAGGCATTC & \\
\hline & FluB-LF & Forward loop & TYAAACGGAACTTCCCTTCTTTC & 23 \\
\hline & FluB-LB & Reverse loop & GGATACAAGTCCTTATYYAACTCTGC & 25 \\
\hline
\end{tabular}

Notes: The polymorphic sites of the primers are underlined; $Y$ represents degenerate $\mathrm{C}$ or $\mathrm{T}$; $\mathrm{R}$ represents degenerate $\mathrm{A}$ or $\mathrm{G}$; $\mathrm{M}$ represents degenerate $\mathrm{A}$ or $\mathrm{C}$. Abbreviations: RT-LAMP, reverse-transcription loop-mediated isothermal amplification; HA, hemagglutinin; MI, matrix protein I; NSI, nonstructural protein I; nt, nucleotide. 
A 757

$\downarrow \mathrm{F} 3 \longrightarrow \quad \mathrm{F} 2$

CCGGGAGACAAAATAACATTCGAAGCAACTGGAAATCTAGTGGTACCGAGATATGCATTCGCAATGGAAAGAAATGCTGGATCTGGTATTA GGCCCTCTGTTTTATTGTAAGCTTCGTTGACCTTTAGATCACCATGGCTCTATACGTAAGCGTTACCTTTCTTTACGACCTAGACCATAAT

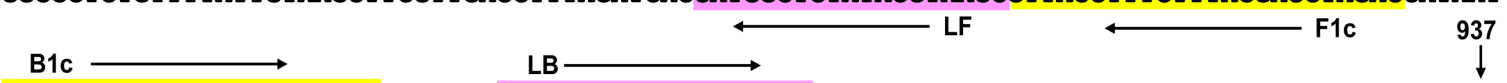

TCATTTCAGATACACCAGTCCACGATTGCAATACAACTTGTCAGACACCCAAGGGTGCTATAAACACCAGCCTCCCATTTCAGAATATAC AGTAAAGTCTATGTGGTCAGGTGCTAACGTTATGTTGAACAGTCTGTGGGTTCCCACGATATTTGTGGTCGGAGGGTAAAGTCTTATATG

B2

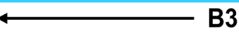

B 425

$\downarrow \mathrm{F} 3 \longrightarrow \quad \mathrm{F} 2$

AGGATGGGGGCTGTAACCACTGAAGTGGCATTTGGCCTGGTATGTGCAACATGTGAGCAGATTGCTGACTCCCAGCACAGGTCTCATAGGC TCCTACCCCCGACATTGGTGACTTCACCGTAAACCGGACCATACACGTTGTACACTCGTCTAACGACTGAGGGTCGTGTCCAGAGTATCCG

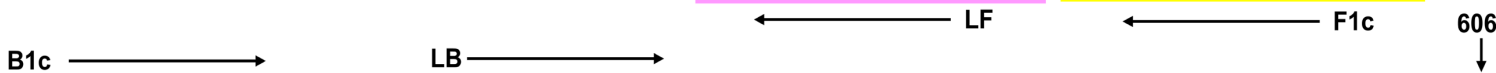

AGATGGTGGCAACAACCAATCCATTAATAAAACATGAGAACAGAATGGTTTTGGCCAGCACTACAGCTAAGGCTATGGAGCAAATGGCTGG TCTACCACCGTTGTTGGTTAGGTAATTATTTTGTACTCTTGTCTTACCAAAACCGGTCGTGATGTCGATTCCGATACCTCGTTTACCGACC

C 442
F3
F2

AGGGACATGAACAACAAAGATGCAAGGCAAAAGATAAAGGAGGAAGTAAACACTCAGAAAGAAGGGAAGTTCCGTTTGACAATAAAAAGGG TCCCTGTACTTGTTGTTTCTACGTTCCGTTTTCTATTTCCTCCTTCATTTGTGAGTCTTTCTTCCCTTCAAGGCAAACTGTTATTTTTCCC LF

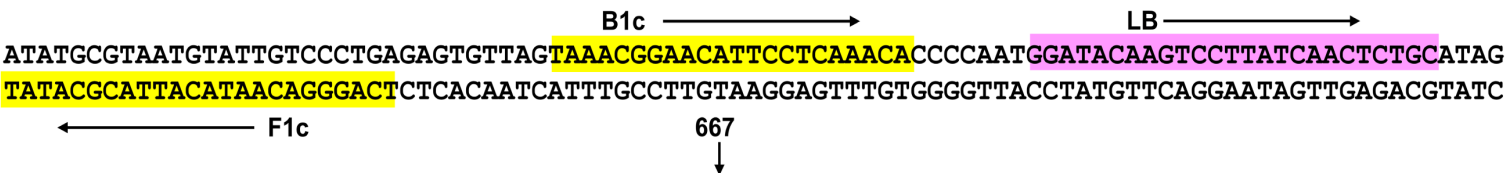

ATTGAATGCATATGACCAGAGTGGAAGGCTTGTTGCTAAACTTG TAACTTACGTATACTGGTCTCACCTTCCGAACAACGATTTGAAC

\section{B2 \\ B3}

Figure I Schematic showing location of multiplex RT-LAMP primer binding sites.

Notes: (A) HA gene of influenza A/HINIpdm09. Assay spans region from nucleotide 757-937 with reference to the HA gene sequence of the $\mathrm{HINIpdm09}$ virus strain $\mathrm{A} /$ /jiangsu/2/2009(HINI). (B) $M /$ gene of influenza $A / H 3 N 2$. Assay spans region from nucleotide $425-606$ with reference to the $M /$ gene sequence of the $H 3 N 2$ virus strain $\mathrm{A} / \mathrm{Nanjing/I/2009}(\mathrm{H} 3 \mathrm{~N} 2)$. (C) NSI gene of influenza B. Assay spans region from nucleotide 442-667 with reference to the NSI gene sequence of the influenza B virus strain $\mathrm{B} / \mathrm{Jiangsu} / 0 \mathrm{I} / 20 \mathrm{I} 5$.

Abbreviations: HA, hemagglutinin; MI, matrix protein I; NSI, nonstructural protein I; RT-LAMP, reverse-transcription loop-mediated isothermal amplification.

no-template control. Twenty-five microliters of mineral oil (Sigma-Aldrich, St Louis, MO, USA) was added to the top of the reaction mixture. $\mathrm{mRT}$-LAMP reactions were run at different temperatures including $60^{\circ} \mathrm{C}, 63^{\circ} \mathrm{C}$ and $65^{\circ} \mathrm{C}$ using a real-time turbidimeter (LA320C; Teramecs, Tokyo, Japan) in order to determine the optimal reaction temperature for obtaining the highest amplification efficiency.

\section{Cascade invasive reaction and AuNPs} hybridization

Three target-specific cascade invasive reactions for each $\mathrm{mRT}$ LAMP reaction were performed in a volume of $20 \mu \mathrm{L}$ containing $1 \times$ reaction buffer $(10 \mathrm{mM} 3-(\mathrm{N}-$ morpholino $)$ propanesulfonic acid, pH 7.5, 0.05\% Tween-20, 0.05\% Nonidet P40 and $7.5 \mathrm{mM} \mathrm{MgCl}_{2}$ ); $0.2 \mu \mathrm{M}$ each of upstream

Table 2 Probes used for cascade invasive reaction and modification of AuNPs

\begin{tabular}{|c|c|c|c|c|}
\hline Target & Name & Type & Sequence $\left(5^{\prime}-3^{\prime}\right)$ & Modification \\
\hline \multirow[t]{2}{*}{$\mathrm{A} / \mathrm{HINIpdm09}$} & $09 \mathrm{HI}-\mathrm{Up}_{\mathrm{p}}$ & $U_{p}$ & ATCTGGTATTATCATTTCAGATACACCAGTT & \\
\hline & $09 \mathrm{HI}-\mathrm{Dp}$ & $D_{p}$ & CGCGCCGAGG CCACGATTGCAATACAAC & \\
\hline \multirow[t]{2}{*}{$\mathrm{A} / \mathrm{H} 3$} & $\mathrm{H} 3-\mathrm{Up}_{\mathrm{p}}$ & $U_{p}$ & GTCTCATAGGCAGATGGTGGCT & \\
\hline & $\mathrm{H} 3-\mathrm{Dp}$ & $\mathrm{Dp}_{\mathrm{p}}$ & CGCGCCGAGG AACAACCAATCCATTAATAAAAC & \\
\hline \multirow[t]{2}{*}{ Influenza B } & FluB-Up & $U_{p}$ & TGTATTGTCCTTGAGAGTGTTGGTAAAT & \\
\hline & FluB-Dp & $D_{p}$ & CGCGCCGAGG CGGAACATTCCTCAAACA & \\
\hline \multirow[t]{4}{*}{ Universal } & $\mathrm{Hp}$ & $\mathrm{Hp}$ & GTCTTGTGGTACTGCACTCGTCTCGGTTTTCCGA & \\
\hline & & & GACGAGTCCTCGGCGCGATCGTGATGAACCAT & \\
\hline & $3^{\prime}-\mathrm{AuNP}$ & nanoparticle probe & GCAGTACCACAAGACAAAAAAAAAA & $3^{\prime}-\mathrm{SH} \mathrm{C6}$ \\
\hline & $5^{\prime}-A u N P$ & nanoparticle probe & AAAAAAAAAAATGGTTCATCACGAT & $5^{\prime}-\mathrm{SH}$ C6 \\
\hline
\end{tabular}

Abbreviations: AuNPs, gold nanoparticles; Up, upstream probe; Dp, downstream probe; Hp, hairpin probe. 
and downstream probes; $0.2 \mu \mathrm{M}$ hairpin probe; $100 \mathrm{ng}$ Archaeoglobus fulgidus flap endonuclease (AfuFEN) enzyme, which was prepared in our lab as described previously; ${ }^{30}$ and $5 \mu \mathrm{L}$ diluted RT-LAMP amplification products $(30 \mu \mathrm{L}$ deionized water was added to $20 \mu \mathrm{L}$ products). The mixtures were then incubated at $85^{\circ} \mathrm{C}$ for 1 min followed by $63^{\circ} \mathrm{C}$ for $20 \mathrm{~min}$. After cascade invasive reaction, $3 \mu \mathrm{L}$ of each previously prepared oligonucleotide probe-modified AuNPs (30 $\mathrm{nM})$ and $5 \mu \mathrm{L}$ of $\mathrm{NaCl}(500 \mathrm{mM})$ were together added into each reaction tube. Hybridization was performed at $55^{\circ} \mathrm{C}$ for $30 \mathrm{~min}$. Then, the result was observed with naked eyes directly or after brief centrifugation $(1,000 \times g$ for $30 \mathrm{~s})$ of the products. The negative reaction mixture became colorless because of the aggregation of AuNPs, while the positive reaction mixture remained red.

\section{Analytical sensitivity and specificity of mRT-LAMP-CIRN}

To evaluate the analytical sensitivity of the mRT-LAMPCIRN assay, synthetic RNA standard for each target was tenfold serially diluted (ranging from $10^{4}$ to $10^{\circ} \mathrm{RNA}$ copies $/ \mu \mathrm{L}$ ) and was used as mRT-LAMP-CIRN template. The specificity of the assay was assessed by analyzing the RNA extracts from influenza viruses A/Jiangsu/2/2009(H1N1), A/Nanjing/1/2009(H3N2) and B/Jiangsu/01/2015, and from each control virus mentioned above separately. RNA extracts from the three influenza virus strains were also tested in different combinations in a single reaction.

\section{Evaluation of the mRT-LAMP-CIRN assay with clinical specimens}

To evaluate the performance characteristics of the mRTLAMP-CIRN assay, 94 clinical nasopharyngeal swab specimens collected from influenza-like cases were analyzed by mRT-LAMP-CIRN assay with the parallel analysis by real-time RT-PCR where primers and probes were all recommended by the WHO. ${ }^{31,32}$ Real-time RT-PCR was performed using the SuperScript ${ }^{\circledR}$ III Platinum One-Step qRT-PCR System (Thermo Fisher Scientific, Carlsbad, CA, USA). The cycling conditions were: $50^{\circ} \mathrm{C}$ for $20 \mathrm{~min}, 95^{\circ} \mathrm{C}$ for $2 \mathrm{~min}$, followed by 40 cycles in $15 \mathrm{~s}$ at $94^{\circ} \mathrm{C}$ and $1 \mathrm{~min}$ at $60^{\circ} \mathrm{C}$.

\section{Results}

\section{Principle of mRT-LAMP-CIRN assay}

The overall process of the mRT-LAMP-CIRN assay includes RT-LAMP amplification of viral nucleic acids, signal amplification by cascade invasive reaction and optical detection using oligonucleotide probe-modified AuNPs. A schematic of the principles of cascade invasive reaction and signal detection with AuNPs is shown in Figure 2. Briefly, the upstream and downstream probes hybridize with RT-LAMP products, forming a one base overlapping structure at the $3^{\prime}$ end of the upstream probe, which can be recognized and cleaved by AfuFEN enzyme. Then the cleaved $5^{\prime}$ flap of the downstream probe hybridizes with the hairpin probe, which can drive a secondary invasive reaction. To visibly detect whether the hairpin probe is cleaved, two types of oligonucleotide probe-modified AuNPs, which can hybridize with the two ends of hairpin probe, are added. If the target of RT-LAMP product is present, the cleaved hairpin probe cannot trigger the aggregation of oligonucleotide probe-modified AuNPs, leading to red color of the reaction. Conversely, if the target of RT-LAMP product is absent and the hairpin probe is intact without cleavage, leading to the aggregation of AuNPs, then the reaction becomes colorless.

\section{Optimization of the mRT-LAMP- CIRN assay}

The real-time turbidimetry device enables observation of LAMP primer kinetics. To optimize the mRT-LAMP conditions, at the commencement of this study, we used a real-time turbidimeter (LA320C; Teramecs, Tokyo, Japan) to determine the optimal primer sequences, primer concentrations, reaction temperature, as well as amplification time. The optimal primer sequences and concentrations, determined using various concentrations of synthetic RNA standards as templates, are shown in Table 1 and described in Materials and methods. The details of the optimization of reaction temperature and amplification time are described in the Supplementary material.

\section{Analytical sensitivity of the mRT- LAMP-CIRN}

The analytical sensitivity of the mRT-LAMP-CIRN assay was evaluated by testing synthetic RNA standards ranging from $10^{4}$ to $10^{0} \mathrm{RNA}$ copies $/ \mu \mathrm{L}$. As shown in Figure $3 \mathrm{~A}-\mathrm{C}$, the mRT-LAMP-CIRN assay could detect $10^{1}$ copies of synthetic RNA for both $\mathrm{A} / \mathrm{H} 1 \mathrm{~N} 1 \mathrm{pdm} 09$ and $\mathrm{A} / \mathrm{H} 3$, and $10^{2}$ copies of synthetic RNA for influenza B. Identical results were obtained on three separate days indicating that the mRTLAMP-CIRN assay was both robust and reproducible. The mRT-LAMP reactions were also monitored by a real-time turbidimeter. As shown in Figure 3D and F, the detection limits for $\mathrm{A} / \mathrm{H} 1 \mathrm{~N} 1 \mathrm{pdm} 09$ and influenza $\mathrm{B}$ were $10^{1}$ and $10^{2}$ copies of synthetic RNA, respectively; these were equivalent to those obtained by mRT-LAMP-CIRN (Figure 3A and C). Nevertheless, the detection limit for $\mathrm{A} / \mathrm{H} 3$ obtained by realtime turbidity detection was an order of magnitude lower than that obtained by mRT-LAMP-CIRN (Figure 3B and E), 

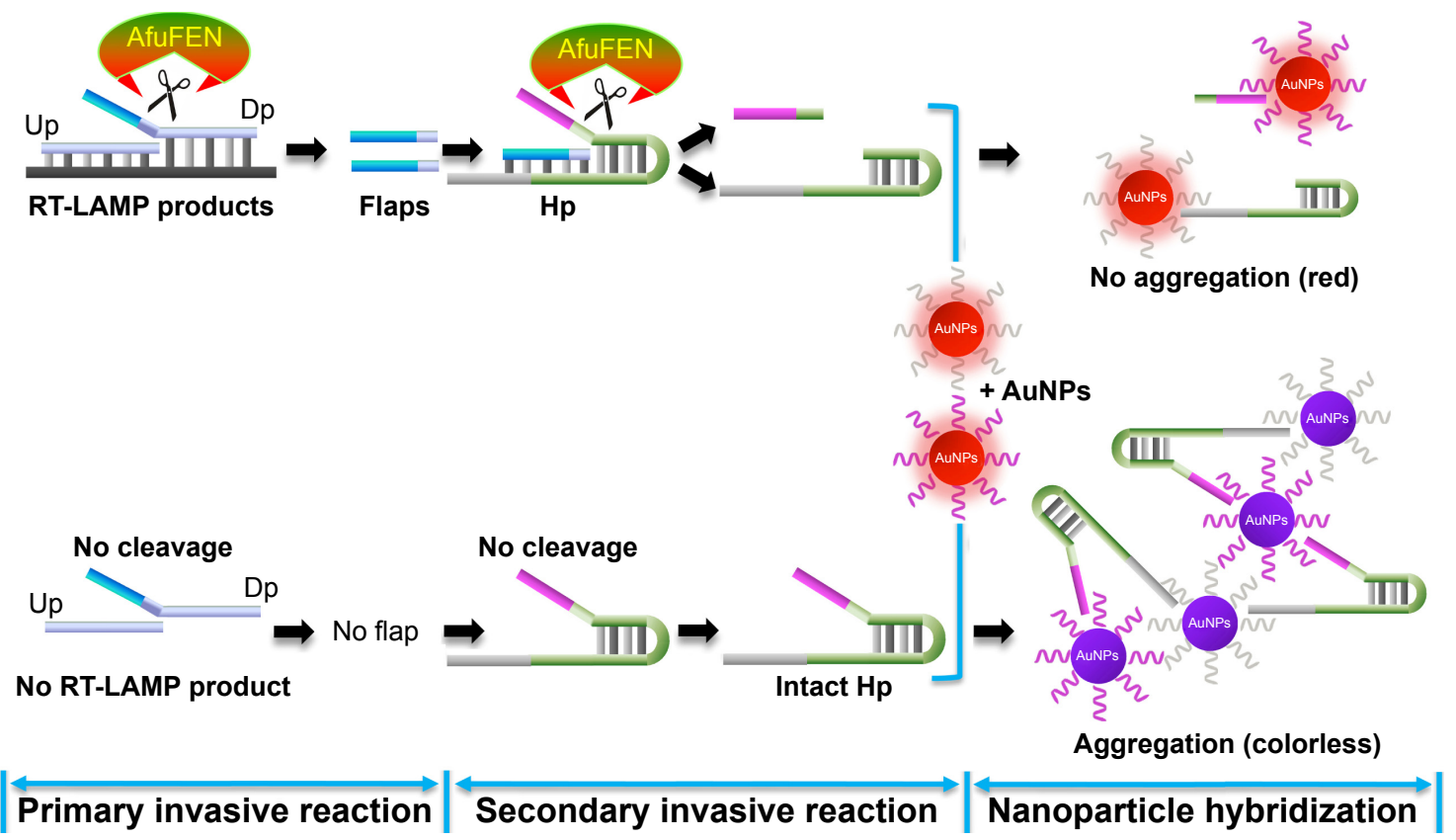

Figure 2 The principles of cascade invasive reaction and signal detection with AuNPs for detecting multiplex RT-LAMP products.

Notes: The system contains three steps: primary invasive reaction, secondary invasive reaction and nanoparticle hybridization. Primary invasive reaction: the Up, Dp and RT-LAMP amplicon form a one-base overlapping structure at the $3^{\prime}$ end of the Up. AfuFEN enzyme specifically recognizes the structure and cleaves the $5^{\prime}$ flap of the Dp. Secondary invasive reaction: the cleaved $5^{\prime}$ flap from the target-specific primary invasive reaction hybridizes with the $\mathrm{Hp}$ and drives a secondary invasive reaction. Then the $\mathrm{Hp}$ is cleaved by AfuFEN. Nanoparticle hybridization: if the target of RT-LAMP product is present, the cleaved Hp cannot trigger the aggregation of AuNPs, resulting in red-colored dispersions of AuNPs. Conversely, if the target of RT-LAMP product is absent, the Hp remains intact without DNA cleavage, yielding nanoparticle aggregationinduced precipitation, and then the reaction mixture becomes colorless.

Abbreviations: Up, upstream probe; Dp, downstream probe; Hp, hairpin probe; AfuFEN, Archaeoglobus fulgidus flap endonuclease; AuNPs, gold nanoparticles; RT-LAMP, reverse-transcription loop-mediated isothermal amplification.

A

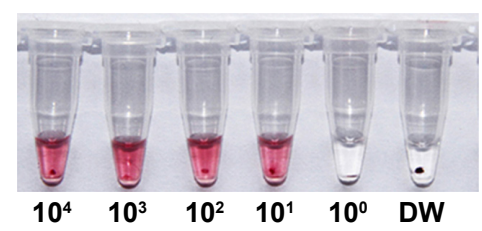

D

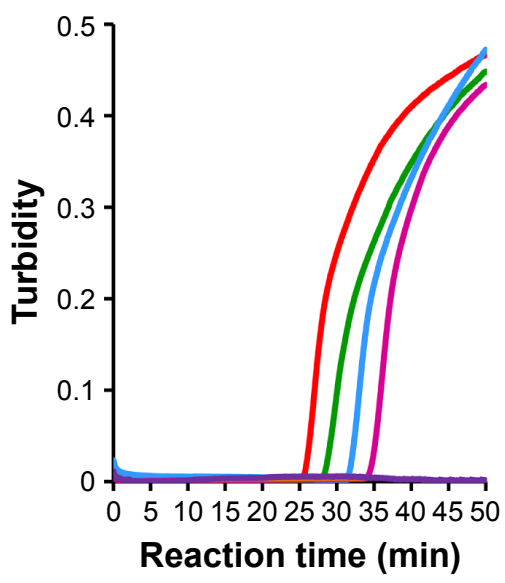

B

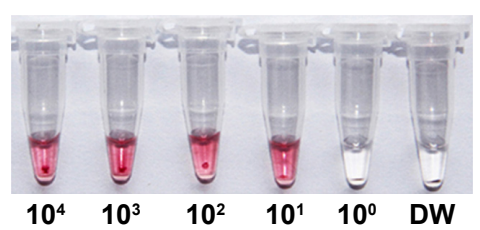

$\mathbf{E}$

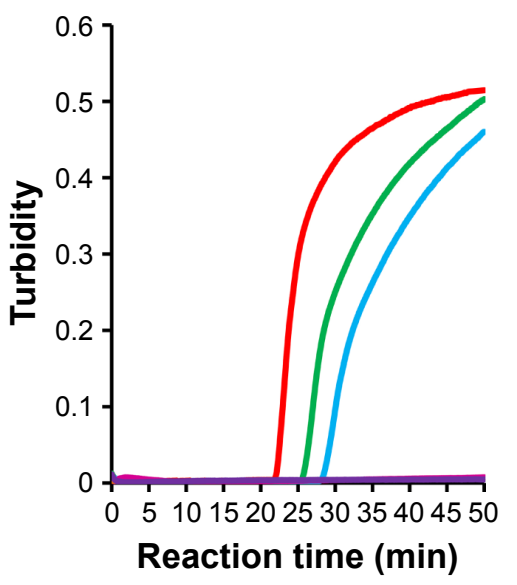

C

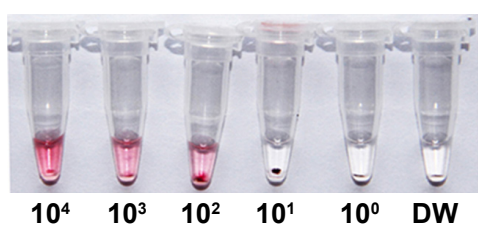

$\mathbf{F}$

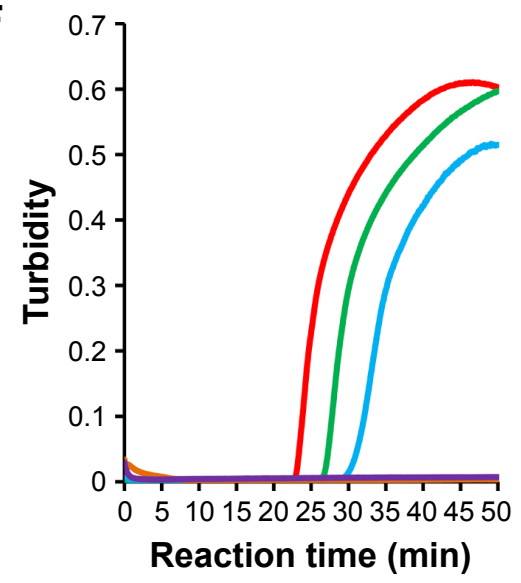

$-10^{4}-10^{3}-10^{2}-10^{1}-10^{0}-\mathrm{DW}$

Figure 3 Sensitivity test results of the mRT-LAMP-CIRN assay for detecting influenza $A / H I N I p d m 09, A / H 3$ and influenza $B$.

Notes: Ten-fold serial dilutions of $\mathrm{A} / \mathrm{HINIpdm09}(\mathbf{A}), \mathrm{A} / \mathrm{H} 3(\mathbf{B})$ and influenza $\mathrm{B}(\mathbf{C}) \mathrm{RNA}$ transcripts (ranging from $10^{4}$ to $10^{\circ} \mathrm{RNA}$ copies) were detected by the mRT-LAMP-CIRN assay. The multiplex RT-LAMP amplification reactions for A/HINIpdm09 (D), A/H3 (E) and influenza B (F) RNA transcripts were also real-time monitored by a turbidimeter and the corresponding curves of concentrations of templates were marked in the figure.

Abbreviations: mRT-LAMP-CIRN, multiplex reverse-transcription loop-mediated isothermal amplification coupled with cascade invasive reaction using nanoparticles as a sensor; DW, distilled water. 
A

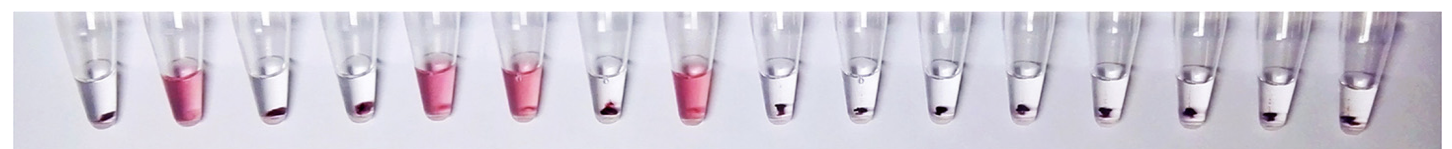

B

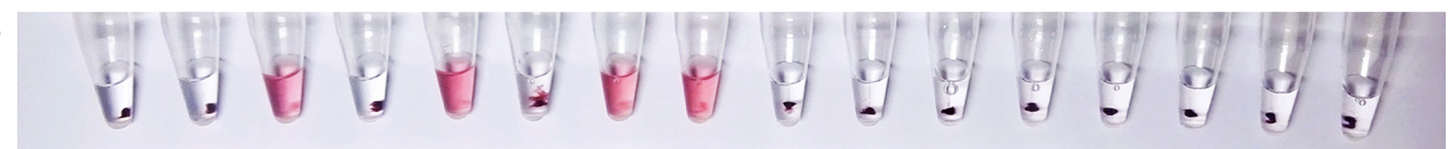

C

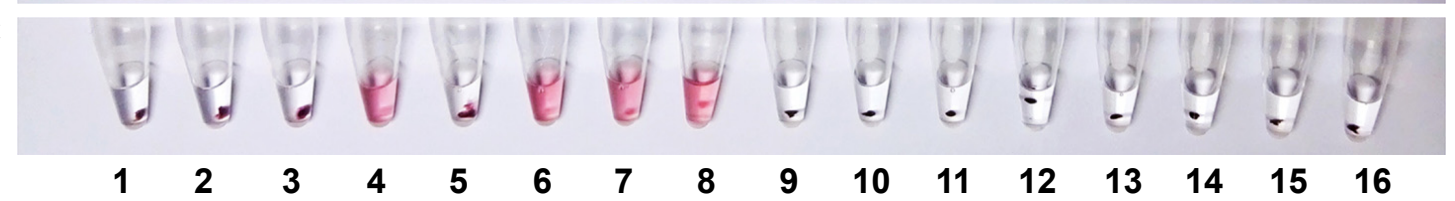

Figure 4 Specificity test results of the mRT-LAMP-CIRN assay for detection of influenza viruses.

Notes: The analytical specificity of the mRT-LAMP-CIRN assay was assessed by testing the RNA extracts from influenza A/HINIpdm09, A/H3N2 and influenza B, and from various control viruses separately. RNA extracts from the three influenza virus strains were also tested in different combinations in a single reaction. (A) The cascade invasive reactions were performed with $09 \mathrm{HI}-\mathrm{Up}_{\mathrm{p}}, 09 \mathrm{HI}-\mathrm{Dp}$ and $\mathrm{Hp}$. (B) The cascade invasive reactions were performed with $\mathrm{H} 3-\mathrm{Up}$, $\mathrm{H} 3-\mathrm{Dp}$ and $\mathrm{Hp}$. (C) The cascade invasive reactions were performed with FluB-Up, FluB-Dp and Hp. I: no-template control; 2: A/HINIpdm09; 3: A/H3N2; 4: influenza B; 5: A/HINIpdm09 and A/H3N2; 6: A/HINIpdm09 and influenza B; 7: A/H3N2 and influenza B; 8: A/HINIpdm09, A/H3N2 and influenza B; 9: A/HINI; I0: A/H5NI; II: A/H7N9; I2: A/H9N2; I3: respiratory syncytial viruses type $A$; 14: respiratory syncytial viruses type $B$; 15: parainfluenza viruses types I; 16: parainfluenza viruses types 2.

Abbreviations: $\mathrm{mRT}$-LAMP-CIRN, multiplex reverse-transcription loop-mediated isothermal amplification coupled with cascade invasive reaction using nanoparticles as a sensor; Up, upstream probe; Dp, downstream probe; Hp, hairpin probe.

indicating that for RT-LAMP product analysis, the sensitivity of cascade invasive reaction using AuNPs as a sensor was higher than real-time turbidity detection.

\section{Analytical specificity of the mRT- LAMP-CIRN}

To evaluate the analytical specificity of the mRT-LAMPCIRN assay, RNA extracts from influenza viruses A/Jiangsu/ 2/2009(H1N1), A/Nanjing/1/2009(H3N2) and B/Jiangsu/ $01 / 2015$, and from various control viruses that could cause similar symptoms were tested separately. RNA extracts from the three influenza virus strains were also tested in different combinations in a single reaction. As shown in Figure 4, for single target detection, the positive reactions with red color in the reaction tubes were only observed in the extracts of influenza $\mathrm{A} / \mathrm{H} 1 \mathrm{~N} 1 \mathrm{pdm} 09, \mathrm{~A} / \mathrm{H} 3 \mathrm{~N} 2$ and influenza $\mathrm{B}$ viruses, whereas all of the control viruses, including the seasonal influenza virus $\mathrm{A} / \mathrm{H} 1 \mathrm{~N} 1$, avian influenza viruses $\mathrm{A} / \mathrm{H} 5 \mathrm{~N} 1$, $\mathrm{A} / \mathrm{H} 7 \mathrm{~N} 9$ and $\mathrm{A} / \mathrm{H} 9 \mathrm{~N} 2$, respiratory syncytial viruses types $\mathrm{A}$ and $\mathrm{B}$, and parainfluenza viruses types 1 and 2 were negative. For multiple target detection, the mRT-LAMP-CIRN assay could differentiate two or three targets in a single reaction successfully (Figure 4). These results demonstrated the high analytical specificity of the mRT-LAMP-CIRN assay.

\section{Clinical performance}

To evaluate the clinical performance characteristics of the mRT-LAMP-CIRN assay, a total of 94 clinical specimens collected from influenza-like cases were analyzed by mRT-LAMP-CIRN assay and by real-time RT-PCR in parallel. As shown in Table 3, out of 58 specimens that were proven positive for influenza viruses by real-time RT-PCR,

Table 3 Clinical performance of mRT-LAMP-CIRN compared with real-time RT-PCR for detecting influenza A/HINIpdm09, A/H3 and influenza $B$

\begin{tabular}{|c|c|c|c|c|}
\hline \multirow[t]{2}{*}{ mRT-LAMP-CIRN } & \multicolumn{2}{|c|}{ Real-time RT-PCR } & \multicolumn{2}{|c|}{ Performance characteristics } \\
\hline & Positive & Negative & Sensitivity, \% & Specificity, \% \\
\hline \multicolumn{5}{|l|}{ A/HINIpdm09 } \\
\hline Positive & 34 & 0 & 97.1 & 100 \\
\hline Negative & 1 & 59 & & \\
\hline \multicolumn{5}{|l|}{$\mathrm{A} / \mathrm{H} 3$} \\
\hline Positive & 14 & 0 & 100 & 100 \\
\hline Negative & 0 & 80 & & \\
\hline \multicolumn{5}{|l|}{ Influenza B } \\
\hline Positive & 9 & 0 & 100 & 100 \\
\hline Negative & 0 & 85 & & \\
\hline \multicolumn{5}{|c|}{ Combined $\mathrm{A} / \mathrm{HIN} / \mathrm{pdm} 09, \mathrm{~A} / \mathrm{H} 3$ and FluB } \\
\hline Positive & 57 & 0 & 98.3 & 100 \\
\hline Negative & I & 36 & & \\
\hline
\end{tabular}

Abbreviations: mRT-LAMP-CIRN, multiplex reverse-transcription loop-mediated isothermal amplification coupled with cascade invasive reaction using nanoparticles as a sensor; RT-PCR, reverse-transcription polymerase chain reaction. 
mRT-LAMP-CIRN detected 34/35 influenza A/H1N1pdm09, 14/14 influenza A/H3 and 9/9 influenza B. Compared to real-time RT-PCR, the sensitivity and specificity of the mRT-LAMP-CIRN assay for detecting influenza A/ H1N1pdm09 were 97.1\% (34/35) and 100\% (59/59), respectively. For detecting influenza $\mathrm{A} / \mathrm{H} 3$, the sensitivity and specificity of the assay were $100 \%(14 / 14)$ and $100 \%$ $(80 / 80)$, and for influenza $B$, the sensitivity and specificity were also $100 \%(9 / 9)$ and 100\% (85/85), respectively. In summary, the mRT-LAMP-CIRN assay had an overall sensitivity and specificity of $98.3 \%(57 / 58)$ and $100 \%(36 / 36)$, respectively.

\section{Discussion}

Since many respiratory viruses, including influenza viruses can cause similar symptoms, it is impossible for clinicians to distinguish one virus from another; therefore, they rely on the laboratory tests to identify the etiologic agent. Considering the morbidity and mortality caused by influenza viruses every year, sensitive and convenient laboratory methods to identify influenza virus subtypes are urgently needed. These are important for initial clinical treatment, avoidance of antibiotic misuse, as well as prevention of influenza virus transmission. In this study, we developed a sensitive mRT-LAMP-CIRN assay for the specific detection of three subtypes of influenza viruses, including influenza A/H1N1pdm09, A/H3 and influenza B. Because no specialized instrument is required, and the read-out can be easily analyzed by visual inspection by operators, the mRT-LAMPCIRN assay described in this study is more suitable for use in low-equipment setting laboratory and on-site testing.

Several isothermal amplification methods have been developed that can pave the way for molecular diagnosis of infectious pathogens in simplified formats. LAMP is a promising method that has been developed to detect various pathogens. ${ }^{11,13-17}$ LAMP amplicons can be determined by agarose gel electrophoresis, ${ }^{33}$ lateral flow dipstick, ${ }^{14}$ turbidity or fluorescence measurement, ${ }^{34,35}$ or even visual inspection of white precipitates or color changes. ${ }^{36,37}$ Due to the properties of cost- and time-saving, multiplex detection represents the development trend of bioassays. Numerous multiplex molecular diagnostic methods such as multiplex PCR/RT-PCR have been developed. ${ }^{38}$ However, for multiplex LAMP, the differentiation of the ladder-like amplicons derived from multiple targets is still challenging today. To achieve multiplex LAMP detection, methods based on either end-point ${ }^{18,19}$ or real-time analysis $^{20-22}$ have been developed to identify the amplicons derived from multiple targets. Nevertheless, all these strategies require specialized and complicated instruments, which hinders the on-site application of multiplex LAMP assay. Invasive reaction is reported to be a highly sensitive and specific DNA detection method that amplifies the targetDNA-specific signal instead of the DNA template. ${ }^{26,27}$ By using invasive reaction probes that were specific for multiplex LAMP amplicons, the ladder-like LAMP products from different targets were successfully differentiated and identified. By taking advantage of cascade invasive reaction and the remarkable optical property of AuNPs in different aggregated states, the mRT-LAMP-CIRN assay developed in this study presents us with an alternative molecular method for sensitive multiplex LAMP product detection without the need for any complicated instruments. Because the mRT-LAMP-CIRN assay amplifies three targets simultaneously in a single tube, the cost for amplification of each target is reduced to onethird of the traditional singleplex LAMP. As the AfuFEN enzyme and AuNPs were prepared in our lab, the total cost of mRT-LAMP-CIRN assay for detecting one target is about two-thirds of traditional LAMP. Moreover, this pilot study may provide a strategy for establishing multiplex LAMP to much more than three plex, so that the cost for detecting each target would be reduced further.

The high analytical sensitivity of LAMP has been reported previously. ${ }^{39}$ Several possible factors may contribute to this fact. For example, the LAMP reaction is less affected by the presence of inhibitory substances in biological materials, less sensitive to various salts, and can tolerate the inhibitory effect of large concentrations of templates. ${ }^{40}$ However, previous studies have shown that due to the mutual interference of multiple primers, the sensitivities of multiplex nucleic acid detection tests tend to decrease. In this study, by coupling cascade invasive reaction with mRT-LAMP, one RT-LAMP amplicon could yield large numbers of cleaved hairpin probes (Figure 2); thus, the analytical sensitivity of mRT-LAMP might be increased by this signal amplification step. Indeed, the sensitivity for A/H3 obtained by mRT-LAMP-CIRN was ten times higher than that obtained by real-time turbidity detection. However, for $10^{\circ}$ copy of A/H1N1pdm09 template and $10^{1}$ copies of influenza B templates (one order of magnitude lower than their detection limits), the mRT-LAMP amplicons might be too limited, or no amplification occurred. So, they also could not be detected by cascade invasive reaction using nanoparticles as a sensor despite the signal amplification effect.

Because LAMP offers highly efficient amplification of target DNA, once the reaction tube is opened after LAMP reaction, there is a risk of aerosol contamination. ${ }^{41}$ 
To overcome this problem, in this study, mineral oil was added to the top of the LAMP reaction mixture to ensure that the amplicons did not disseminate into the air when the reaction tubes were opened. To further reduce the chances of contamination, protocols similar to those followed for PCR were adopted in this study. For example, the sample treatment, solution preparation and amplicon identification were carried out in separate areas.

The amplification mechanism of LAMP confers on the mRT-LAMP-CIRN assay inherent specificity because eight regions of each target sequence must be recognized by the four sequence-specific primers and two loop primers for amplification to occur. In addition, the invasive reaction is a specific DNA detection method because the mRT-LAMP amplicon must contain a sequence to which the invasive reaction upstream and downstream probes will hybridize in order to produce a positive result. By combining the specificities of both LAMP and cascade invasive reaction, the mRT-LAMP-CIRN assay developed in this study should provide higher specificity in theory. Our results showed that the mRT-LAMP-CIRN assay had 100\% analytical specificity for identification of influenza A/H1N1pdm09, A/H3N2 and influenza $\mathrm{B}$ viruses, and there was no cross-reaction with other genetically or clinically related control viruses tested in this study.

As real-time RT-PCR has been widely accepted as the standard method for detecting influenza virus RNA, during clinical specimen detection, we compared the new assay described in this study with real-time RT-PCR. The results showed that, compared to real-time RT-PCR, the mRT-LAMP-CIRN assay had an overall sensitivity and specificity of $98.3 \%$ and $100 \%$, respectively. Out of 35 specimens that were positive for influenza A/H1N1pdm09 as detected by real-time RT-PCR, 34 were determined to be positive by mRT-LAMP-CIRN. The difference in number detected may be due to the fact that eight regions of the target sequence must be recognized by the six primers in $\mathrm{mRT}$-LAMP reaction, while only three regions of the target sequence need to be recognized in real-time RTPCR reaction. Because of the frequent mutation of influenza virus genome, there might be a base variation in this specimen that affected the detection effect of mRT-LAMP-CIRN but not real-time RT-PCR. Further testing on additional clinical specimens is still needed to evaluate the clinical performance characteristics of this mRT-LAMP-CIRN assay.

In conclusion, a highly sensitive and specific mRTLAMP-CIRN assay has been developed for identification of influenza viruses, including influenza A/H1N1pdm09, A/H3 and influenza B. Cost savings and no need for any complicated instruments make this assay more suitable for on-site use and low-equipment setting laboratory use.

\section{Acknowledgments}

The study was supported in part by the Important National Science \& Technology Specific Projects during the 25-year plan period (2013ZX10004103-006), Jiangsu Province Science \& Technology Demonstration Project for Emerging Infectious Diseases Control and Prevention (No BE2015714), the National Natural Science Foundation of China (81501785, 81601732, 61401217), the Natural Science Foundation of Jiangsu Province (BK20141030, BK20161583, BK20140900), the key discipline of Jiangsu Province (ZDXKA2016008) and the Young Medical Talent Project of Jiangsu Province (QNRC2016537).

\section{Disclosure}

The authors report no conflicts of interest in this work.

\section{References}

1. Simonsen L, Spreeuwenberg P, Lustig R, et al. Global mortality estimates for the 2009 Influenza Pandemic from the GLaMOR project: a modeling study. PLoS Med. 2013;10(11):e1001558.

2. Medina RA, Garcia-Sastre A. Influenza A viruses: new research developments. Nat Rev Microbiol. 2011;9(8):590-603.

3. Richard M, Fouchier RA. Influenza A virus transmission via respiratory aerosols or droplets as it relates to pandemic potential. FEMS Microbiol Rev. 2016;40(1):68-85.

4. Molinari NA, Ortega-Sanchez IR, Messonnier ML, et al. The annual impact of seasonal influenza in the US: measuring disease burden and costs. Vaccine. 2007;25(27):5086-5096.

5. Girard MP, Cherian T, Pervikov Y, Kieny MP. A review of vaccine research and development: human acute respiratory infections. Vaccine. 2005;23(50):5708-5724.

6. Ito M, Nukuzuma S, Sugie M, et al. Detection of pandemic influenza A (H1N1) 2009 virus RNA by real-time reverse transcription polymerase chain reaction. Pediatr Int. 2012;54(6):959-962.

7. Suwannakarn K, Payungporn S, Chieochansin T, et al. Typing (A/B) and subtyping $(\mathrm{H} 1 / \mathrm{H} 3 / \mathrm{H} 5)$ of influenza $\mathrm{A}$ viruses by multiplex real-time RT-PCR assays. J Virol Methods. 2008;152(1-2):25-31.

8. Choi JH, Kim MS, Lee JY, et al. Development and evaluation of multiplex real-time RT-PCR assays for seasonal, pandemic A/H1pdm09 and avian A/H5 influenza viruses detection. J Microbiol. 2013;51(2): $252-257$.

9. Munro SB, Kuypers J, Jerome KR. Comparison of a multiplex real-time PCR assay with a multiplex Luminex assay for influenza virus detection J Clin Microbiol. 2013;51(4):1124-1129.

10. Notomi T, Okayama H, Masubuchi H, et al. Loop-mediated isothermal amplification of DNA. Nucleic Acids Res. 2000;28(12):E63.

11. Parida M, Sannarangaiah S, Dash PK, Rao PV, Morita K. Loop mediated isothermal amplification (LAMP): a new generation of innovative gene amplification technique; perspectives in clinical diagnosis of infectious diseases. Rev Med Virol. 2008;18(6):407-421.

12. Whiting SH, Champoux JJ. Properties of strand displacement synthesis by Moloney murine leukemia virus reverse transcriptase: mechanistic implications. J Mol Biol. 1998;278(3):559-577.

13. Huang XY, Hu XN, Ma H, et al. Detection of new bunyavirus RNA by reverse transcription-loop-mediated isothermal amplification. J Clin Microbiol. 2014;52(2):531-535. 
14. Ge Y, Wu B, Qi X, et al. Rapid and sensitive detection of novel avianorigin influenza A (H7N9) virus by reverse transcription loop-mediated isothermal amplification combined with a lateral-flow device. PLoS One. 2013;8(8):e69941.

15. Wang X, Zhu JP, Zhang Q, et al. Detection of enterovirus 71 using reverse transcription loop-mediated isothermal amplification (RT-LAMP). J Virol Methods. 2012;179(2):330-334.

16. Shigemoto N, Fukuda S, Takao S, et al. [Rapid detection of novel influenza A virus and seasonal influenza A (H1N1, H3N2) viruses by reverse transcription-loop-mediated isothermal amplification (RT-LAMP)]. Kansenshogaku Zasshi. 2010;84(4):431-436. Japanese.

17. Curtis KA, Rudolph DL, Owen SM. Rapid detection of HIV-1 by reverse-transcription, loop-mediated isothermal amplification (RT-LAMP). J Virol Methods. 2008;151(2):264-270.

18. Liang C, Chu Y, Cheng S, et al. Multiplex loop-mediated isothermal amplification detection by sequence-based barcodes coupled with nicking endonuclease-mediated pyrosequencing. Anal Chem. 2012; 84(8):3758-3763.

19. Iseki H, Alhassan A, Ohta N, et al. Development of a multiplex loopmediated isothermal amplification (mLAMP) method for the simultaneous detection of bovine Babesia parasites. J Microbiol Methods. 2007;71(3):281-287.

20. Mahony J, Chong S, Bulir D, Ruyter A, Mwawasi K, Waltho D. Multiplex loop-mediated isothermal amplification (M-LAMP) assay for the detection of influenza $\mathrm{A} / \mathrm{H} 1, \mathrm{~A} / \mathrm{H} 3$ and influenza $\mathrm{B}$ can provide a specimen-to-result diagnosis in 40 min with single genome copy sensitivity. J Clin Virol. 2013;58(1):127-131.

21. Tanner NA, Zhang Y, Evans TC Jr. Simultaneous multiple target detection in real-time loop-mediated isothermal amplification. Biotechniques. 2012;53(2):81-89.

22. Wang Y, Wang Y, Lan R, et al. Multiple endonuclease restriction realtime loop-mediated isothermal amplification: a novel analytically rapid, sensitive, multiplex loop-mediated isothermal amplification detection technique. J Mol Diagn. 2015;17(4):392-401.

23. Xu W, Xue X, Li T, Zeng H, Liu X. Ultrasensitive and selective colorimetric DNA detection by nicking endonuclease assisted nanoparticle amplification. Angew Chem Int Ed Engl. 2009;48(37):6849-6852.

24. Xu W, Xie X, Li D, Yang Z, Li T, Liu X. Ultrasensitive colorimetric DNA detection using a combination of rolling circle amplification and nicking endonuclease-assisted nanoparticle amplification (NEANA). Small. 2012;8(12):1846-1850.

25. Zou B, Cao X, Wu H, et al. Sensitive and specific colorimetric DNA detection by invasive reaction coupled with nicking endonucleaseassisted nanoparticles amplification. Biosens Bioelectron. 2015; $66: 50-54$

26. Lyamichev VI, Kaiser MW, Lyamicheva NE, et al. Experimental and theoretical analysis of the invasive signal amplification reaction. Biochemistry. 2000;39(31):9523-9532.

27. Hall JG, Eis PS, Law SM, et al. Sensitive detection of DNA polymorphisms by the serial invasive signal amplification reaction. Proc Natl Acad Sci U S A. 2000;97(15):8272-8277.
28. Fronhoffs $\mathrm{S}$, Totzke $\mathrm{G}$, Stier $\mathrm{S}$, et al. A method for the rapid construction of cRNA standard curves in quantitative real-time reverse transcription polymerase chain reaction. Mol Cell Probes. 2002;16(2):99-110.

29. Hill HD, Mirkin CA. The bio-barcode assay for the detection of protein and nucleic acid targets using DTT-induced ligand exchange. Nat Protoc. 2006;1(1):324-336.

30. Song Q, Qi X, Jia H, et al. Invader assisted enzyme-linked immunosorbent assay for colorimetric detection of disease biomarkers using oligonucleotide probe-modified gold nanoparticles. J Biomed Nanotechnol. 2016;12(4):831-839.

31. WHO.int [homepage on the Internet]. CDC protocol of realtime RTPCR for influenza A(H1N1). Available from: http://www.who.int/csr/resources/ publications/swineflu/CDCRealtimeRTPCR_SwineH1Assay2009_20090430.pdf. Accessed January 6, 2017.

32. WHO.int [homepage on the Internet]. WHO information for molecular diagnosis of influenza virus in humans - update. Available from: http:// www.who.int/influenza/resources/documents/molecular_diagnosis_ influenza_virus_humans_update_201108.pdf. Accessed January 6, 2017.

33. Sun J, Li X, Zeng H, et al. Development and evaluation of loop-mediated isothermal amplification (LAMP) for the rapid diagnosis of Penicillium marneffei in archived tissue samples. FEMS Immunol Med Microbiol. 2010;58(3):381-388.

34. Mori Y, Kitao M, Tomita N, Notomi T. Real-time turbidimetry of LAMP reaction for quantifying template DNA. J Biochem Biophys Methods. 2004;59(2):145-157.

35. Nyan DC, Swinson KL. A novel multiplex isothermal amplification method for rapid detection and identification of viruses. Sci Rep. 2015; 5:17925.

36. Tomita N, Mori Y, Kanda H, Notomi T. Loop-mediated isothermal amplification (LAMP) of gene sequences and simple visual detection of products. Nat Protoc. 2008;3(5):877-882.

37. Zhang XJ, Sun Y, Liu L, Belak S, Qiu HJ. Validation of a loop-mediated isothermal amplification assay for visualised detection of wild-type classical swine fever virus. J Virol Methods. 2010;167(1):74-78.

38. Krause JC, Panning M, Hengel H, Henneke P. The role of multiplex PCR in respiratory tract infections in children. Deutsches Arztebl Int. 2014;111(38):639-645

39. Wang C, Shen X, Lu J, Zhang L. Development of a reverse transcriptionloop-mediated isothermal amplification (RT-LAMP) system for rapid detection of HDV genotype 1. Lett Appl Microbiol. 2013;56(3): 229-235.

40. Kaneko H, Kawana T, Fukushima E, Suzutani T. Tolerance of loopmediated isothermal amplification to a culture medium and biological substances. J Biochem Biophys Methods. 2007;70(3):499-501.

41. Mao Z, Qiu Y, Zheng L, Chen J, Yang J. Development of a visual loop-mediated isothermal amplification method for rapid detection of the bacterial pathogen Pseudomonas putida of the large yellow croaker (Pseudosciaena crocea). J Microbiol Methods. 2012;89(3):179-184. 


\section{Supplementary materials \\ UV spectrum and TEM images of oligonucleotide probe-modified AuNPs}

The normalized optical absorption spectrum for the gold nanoparticles (AuNPs) after modification with oligonucleotide probes is shown in Figure S1A. We found that the oligonucleotide probe-modified AuNPs exhibited a ultraviolet (UV) peak at $530 \mathrm{~nm}$. The Transmission electron microscopy (TEM) images of the free and aggregated AuNPs are shown in Figure S1B and C.

\section{Optimization of the mRT-LAMP-CIRN assay}

RNA extracts from three influenza viral isolates: A/ Jiangsu/2/2009(H1N1), A/Nanjing/1/2009(H3N2) and
$\mathrm{B} / \mathrm{Jiangsu} / 01 / 2015$ were used as templates to optimize the multiplex reverse-transcription loop-mediated isothermal amplification (mRT-LAMP) conditions. The mRT-LAMP reactions were run at three different temperatures including $60^{\circ} \mathrm{C}, 63^{\circ} \mathrm{C}$, and $65^{\circ} \mathrm{C}$ to determine the optimal reaction temperature for obtaining the highest amplification efficiency. At $63^{\circ} \mathrm{C}$, the shortest amplification time and best amplification efficiency were obtained for all the three targets (Figure S2), and thus $63^{\circ} \mathrm{C}$ was chosen as the optimal temperature for all subsequent multiplex RT-LAMP reactions. According to the amplification curves obtained by real-time turbidimetry device, 50 min was chosen as the standard assay time because almost all the reactions had reached the plateau phase of amplification and longer reaction time yielded similar results (Figure S2; Figure 3).

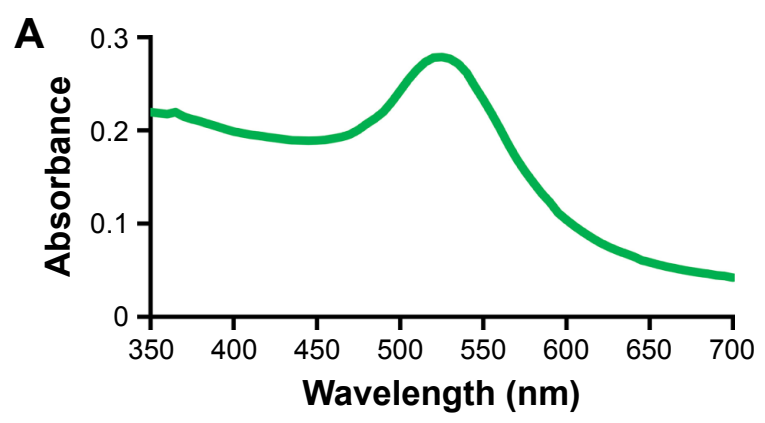

B

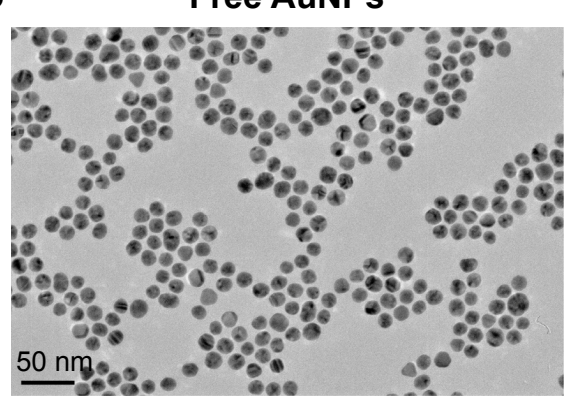

C
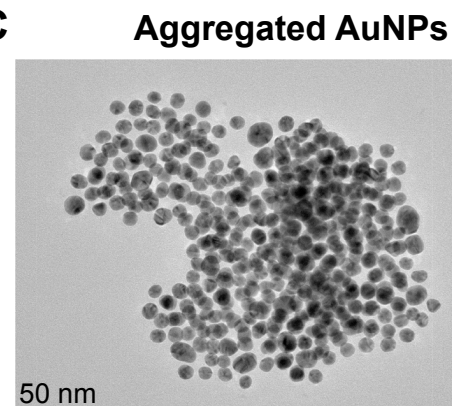

Figure SI Characterization of oligonucleotide probe-modified AuNPs.

Notes: (A) The normalized optical absorption spectrum for the AuNPs after modification with oligonucleotide probes. The TEM images of the free (B) and aggregated (C) AuNPs. Scale bar: $50 \mathrm{~nm}$.

Abbreviations: AuNPs, gold nanoparticles; TEM, transmission electron microscopy. 

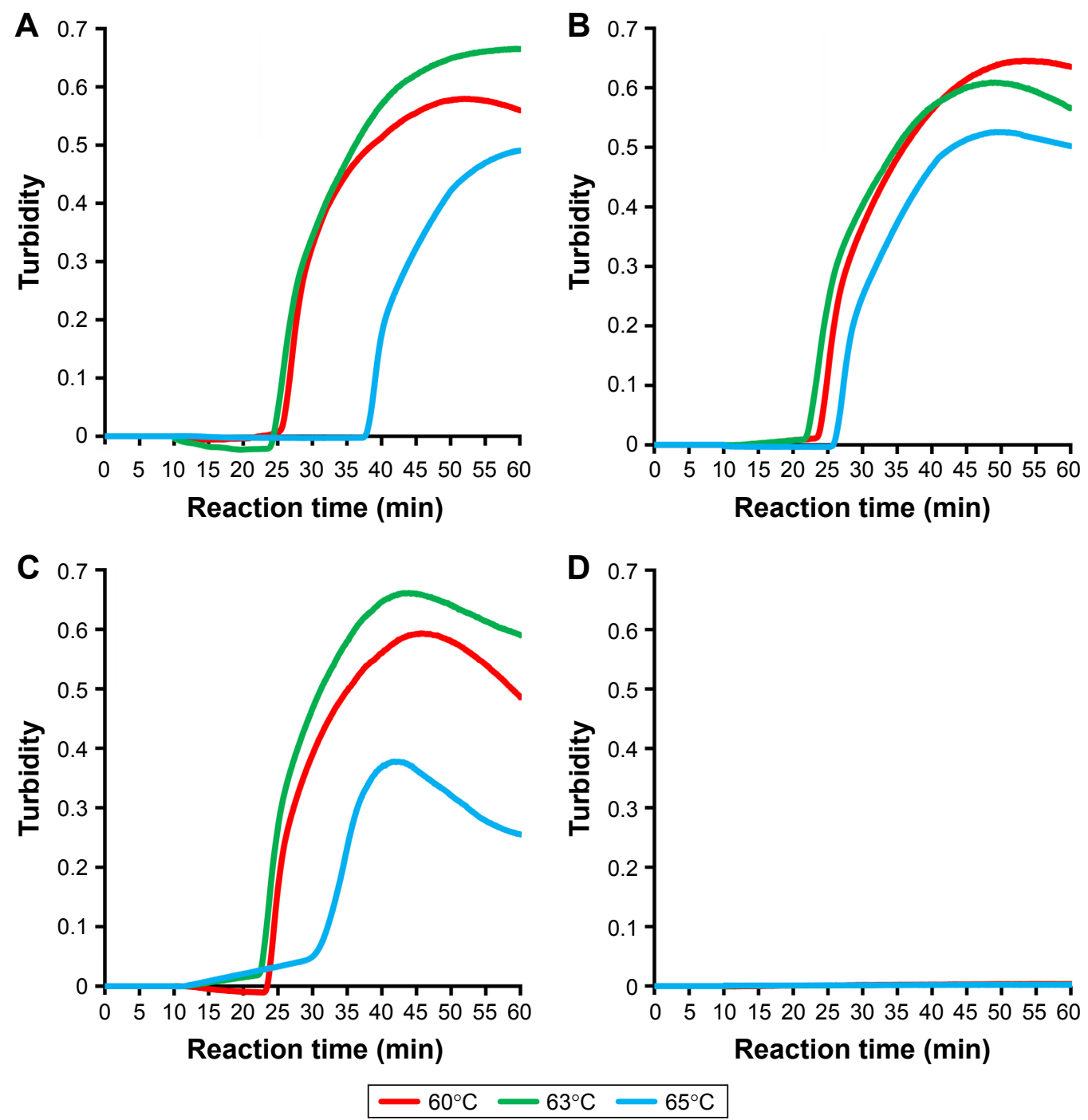

Figure S2 Optimization of the mRT-LAMP-CIRN assay.

Notes: RNA extracts from A/Jiangsu/2/2009(HINI) (A), A/Nanjing/I/2009(H3N2) (B) and B//iangsu/0I/20I5 (C) were used as templates to optimize the mRT-LAMP conditions. Distilled water was used as no-template control (D). The mRT-LAMP reactions were run at $60^{\circ} \mathrm{C}, 63^{\circ} \mathrm{C}$, and $65^{\circ} \mathrm{C}$ for 60 min to determine the optimal reaction temperature and amplification time.

Abbreviation: mRT-LAMP-CIRN, multiplex reverse-transcription loop-mediated isothermal amplification coupled with cascade invasive reaction using nanoparticles as a sensor.

\section{Publish your work in this journal}

The International Journal of Nanomedicine is an international, peerreviewed journal focusing on the application of nanotechnology in diagnostics, therapeutics, and drug delivery systems throughout the biomedical field. This journal is indexed on PubMed Central, MedLine, CAS, SciSearch $®$, Current Contents ${ }^{\circledR} /$ Clinical Medicine,
Journal Citation Reports/Science Edition, EMBase, Scopus and the Elsevier Bibliographic databases. The manuscript management system is completely online and includes a very quick and fair peer-review system, which is all easy to use. Visit http://www.dovepress.com/ testimonials.php to read real quotes from published authors. 